\title{
Article \\ Explicit Formula for Average Run Length of Double Moving Control Chart for INAR(1) Processes
}

\author{
Suganya Phantu, Saowanit Sukparungsee * and Yupaporn Areepong \\ Department of Applied Statistics, Faculty of Applied Science, King Mongkut's University of Technology \\ North Bangkok, 1518 Pracharat 1 road, Wongsawang, Bangsue, Bangkok 10800, Thailand; \\ marchmello_aroy@hotmail.com (S.P.); yupaporn.a@sci.kmutnb.ac.th (Y.A.) \\ * Correspondence: saowanit.s@sci.kmutnb.ac.th; Tel.: +66-917-892-654
}

\begin{abstract}
Count data are used in many fields of practice, especially Poisson distribution as a popular choice for the marginal process distribution. If these counts exhibit serial dependence, a popular approach is to use a Poisson INAR(1) model to describe the autocorrelation structure of process. In this paper, the explicit formulas are proposed to evaluate performance characteristics of Double Moving Average control chart (DMA) for Integer valued autoregressive of serial dependence Poisson process. The characteristics of the control chart are frequently measured as Average Run Length (ARL) which means that the average of observations are taken before a system is signaled to be out-of-control. These proposed explicit formulas of ARL are simple and easy to implement for practitioner. The numerical results show that the DMA chart performs better than others when the magnitudes of shift are moderate and large.
\end{abstract}

Keywords: average run length; double moving average control chart; Poisson count process

\section{Introduction}

Statistical quality control (SQC) is often implemented with processes of counts. Count data occurs in various fields of practice due to the ease of data collection. Among others, application for statistical quality control (counts of nonconformities), to epidemiology (count of cases of a certain disease), or to economy (counts of price change). The marginal distribution of count processes can often be modeled by Poisson distribution, where $\lambda$ denotes the average of the Poisson distribution (Harris [1]). One particular area where these counts can be useful is in process monitoring to detect shifts of a process from an in-control state to various out-of-control states. Hence, quality loss can be reduced or prevented through corrective actions to put the process back into a normal state. Let $N_{t}$ be a process of count data, which is assumed to be stationary with Poisson distributed marginals in the state of statistical control (Wei $\beta$ [2]). Most prominent are two charts of Shewhart type, namely c chart and $\mathrm{u}$ chart, which both monitor the marginal distribution of the process $\left(_{N_{t}}\right)$. For a detailed description, consult Montgomery [3]. Often, the c chart has been used to monitor count data. Although originally developed for independent count data which has also been discussed in previous literature. The Shewhart control chart uses only the information in the last sample and ignores information given by the entire data sequence. Thus, the $\mathrm{c}$ chart is known to have poor performance when detecting small shift in process mean. In the past few decades, Exponentially Weighted Moving Average control chart (EWMA) was introduced by Robert [4]. It is an effective chart to detect small and moderate shifts. Cumulative Sum control chart (CUSUM) was introduced by Page [5]. It is sensitive to small shifts for process mean. Recently, Moving Average control chart (MA) was proposed (see [3]). Khoo [6] studied MA charts to monitor the fraction of non-conforming observations and showed that the MA chart was more efficient than the p chart. Later, Michael et.al. [7] proposed the Double Moving Average Control Chart (DMA) when observation are of normal distribution. The numerical results showed that the DMA chart improved average run length of MA chart by using a Monte Carlo (MC) simulation. Mostly, the count process assumes that the count data 
are independent and identically distributed (i.i.d.). However, observations could be serially autocorrelated which may adversely affect the performance of the control charts under this assumption of independence (Brockwell and Davis [8]). A popular class of models for stationary real valued processes are the autoregressive moving average (ARMA) models. These models have a simple autocorrelation structure and other attractive properties. Because the typical mathematical operations are well defined for count, the recursion ARMA model cannot be applied to the integer valued case, since the multiplication of an integer by a real number usually results in a non-integer valued. This motivates us to replace the scalar multiplication in the recursion ARMA model by binomial thinning (McKenzie [9]). Al.Osh and Alzaid [10] introduced the first order integer-valued autoregressive (INAR(1)) model. It is well suited to model the autocorrelation structure of process with Poisson distribution. The statistical properties of INAR(1) are discussed in McKenzie [11].

The performance of a control chart when the process is in-control can usually be characterized by the in-control Average Run Length (ARLo). It is the average of observation before the control chart gives a false alarm as the in-control process has gone to the out-of-control process. The performance of a control chart when the process is out-of-control is Average of Delay Time (ADT). It is the average of observation between process goes out-of-control and control chart giving an alarm that the process has gone out-of-control. Ideally, the value of ARLo of an acceptable chart should be sufficiently large and the value of ADT should be minimal. Most work focuses on evaluating the ARL0 and ADT for control charts have been studied in previous literature. A basic approach that is often used to test other methods is Monte Carlo (MC) simulation. Roberts [4] studied the ARL for EWMA charts by using simulations for processes following a normal distribution which could be used to find the ARL for a variety of parameter values. Crowder [12] studied numerical quadrature methods to solve the exact Integral Equations (IE) for the ARL for normal distribution. Brook and Evans [13] used an approximate formula for the ARL of EWMA chart by using a finite-state Markov Chain Approach (MCA). Areepong and Novikov [14] derived explicit formulas for ARL of Exponentially Weighted Moving Average control charts. Areepong and Sukparungsee [15] studied an analytical ARL of Binomial double moving average chart. Areepong [16] studied explicit formulas of average run length for a moving average control chart for monitoring the number of defective products. Areepong and Sukparungsee studied [17] studied closed form formulas of average run length of moving average control chart for nonconforming for zero-inflated process. Sukparungsee [18] studied run length of double moving average control chart for zero-inflated count processes. Sukparungsee and Areepong [19] studied explicit expression for the average run length of double moving average scheme for zero-inflated binomial process. Recently, Phantu et.al. [20] studied Explicit expressions of average run length of moving average control chart for Poisson integer valued autoregressive model. In the literature one can find at least four numerical procedures to evaluate average run length. Monte Carlo (MC) is simple to program and based on a large number of sample trajectories, so it is time consuming to run. Moreover, it is difficult to use for optimization, though it is convenient to control accuracy of analytically approximations. Integral Equation (IE) is the most advanced method currently available but it requires intensive programming to implement, even for the case of Gaussian distribution and also for the continuous observations. The Markov Chain Approach (MCA) is considered a popular technique. It is based on the approximation of matrix inversions. In addition, there are no theoretical results on accuracy for this procedure in terms of rate of convergence. The Martingale approach is simple and convenient for approximation but it could also be implemented for the case of light-tailed distributions or the moment generating function of exits. However, the results for average run length and average delay time usually cannot be obtained analytically and intensive programming or specialized software is required to obtain analytical results even for the case of the normal distribution.

In this paper we propose an explicit formula to evaluate ARL and ADT of double moving average control chart (DMA) when observations are Poisson count process. The results show that the performance of a DMA chart is good when the magnitudes of shift are moderate and large.

\section{Methodology}




\subsection{Binomial Thinning}

The binomial thinning operator introduced by Steutel and Harn [21] preserves the status of an integer random variable when $N$ operates on by a parameter $\alpha \in(0,1)$, which is proven to be an adequate alternative to scalar multiplication. If $N$ is a discrete random variable with range $\{0, \ldots, \mathrm{n}\}$. The thinning operation defined as

$$
\alpha \circ N=\sum_{i=1}^{N} X_{i}
$$

where $X_{i}$ are i.i.d. Bernoulli counting sequence random variables $\mathrm{P}\left(X_{i}=1\right)=\alpha$ and $\mathrm{P}\left(X_{i}=0\right)=1-\alpha$. The operator is a random operator and the random variable $\alpha \circ N$ has a binomial distribution with parameters $N$ and $\alpha$ and counts the number of survivors from the count $N$ remaining after thinning. Notice that the thinning operator confers greater dispersion on the number of survivors than the ordinary multiplication operator. For instance in integer time series models, $N$ may often be an equi-dispersed Poisson random variable with equal mean and variance $\lambda$.

Suppose $N_{t-1}$ is an integer random variable arising at time $t-1$ and subjected to binomial thinning to produce the number of survivors in the next period. Then, conditional on $N_{t-1}$ for $\alpha \circ N$ is an integer random variable with variance $\alpha(1-\alpha) \mathrm{N}_{t-1}$, whereas $\alpha \mathrm{N}_{t-1}$ has zero conditional variance (the unconditional counterparts are $\alpha \lambda$ and $\alpha \lambda^{2}$ ). Expectation and variance of $\alpha \circ N$ can be easily obtained by applying well-known rules for conditional moment as follows

$$
E[\alpha \circ N]=\alpha E[N] \quad V[\alpha \circ N]=\alpha^{2} V[N]+\alpha(1-\alpha) E[N] .
$$

It is the first result which justifies to replace the scalar multiplication in usual ARMA models by the probabilistic operation of binomial thinning.

\subsection{Integer Autoregressive Model}

The first integer-valued ARMA model, the INAR(1) model was introduced by McKenzie [9]. It is based on a probabilistic operation of binomial thinning. Alzaid and Al-Osh [10] derived a number of important statistical properties of these models, which are the discrete analogue of the usual AR(1) model. The INAR(1) process is defined by the recursion

$$
N_{t}=\alpha \circ N_{t-1}+\varepsilon_{t}
$$

where ( $\circ$ ) is the thinning operations at time $t$ are performed independently of each other and $\varepsilon_{t}$ and where $N_{t}$ is the observable count at time $t$ and the innovations $\varepsilon_{t}$ are i.i.d. count data. The INAR(1) model is the best fitting model for Poisson marginal. If $\varepsilon_{t}$ follows the Poisson distribution with mean $\mu(1-\alpha)$ then $\varepsilon_{t}$ was $\operatorname{Poi}(\lambda(1-\alpha))$ distribution if the initial count $N_{0}$ is distributed as $\operatorname{Poi}(\lambda)$. Then $N_{t}$ is stationary and distributed as $\operatorname{Poi}(\lambda)$. According to the above situation, it can be modeled as a Poisson INAR(1) model. The expectation and variance of INAR(1) model are

$$
E\left[N_{t}\right]=V\left[N_{t}\right]=\frac{\mu}{1-\alpha}
$$

\subsection{The Double Moving Average Control Chart}

A double moving average control chart (DMA) was proposed by Michael and Khoo [7] and the explicit formula binomial DMA chart of ARL was presented by Areepong and Sukparungsee [19]. The DMA statistic is defined by 


$$
D M A_{i}=\left\{\begin{array}{l}
\frac{M A_{i}+M A_{i-1}+M A_{i-2}+\ldots}{i} ; i \leq w \\
\frac{M A_{i}+M A_{i-1}+\ldots+M A_{i-w+1}}{w} ; w<i<2 w-1, \\
\frac{M A_{i}+M A_{i-1}+\ldots+M A_{i-w+1}}{w} ; i \geq 2 w-1
\end{array}\right.
$$

where $M A_{i}$ is statistic of moving average control chart. Suppose individual observations, $N_{1}, N_{2}, \ldots$, are collected moving average of width $w$ at time $i$ is defined as (Montgomery [3])

$$
M A_{i}=\frac{N_{i}+N_{i-1}+\ldots+N_{i-w+1}}{w}
$$

for period $i \geq w$. For period $i<w$, we do not have $w$ observations to calculate a moving average of width $w$. For these periods the average of all observations up to period $i$ defines the moving average. The mean and variance of double moving average control chart,

$$
E\left[D M A_{i}\right]=\frac{\mu}{1-\alpha}
$$

and

$$
V\left[D M A_{i}\right]=\left\{\begin{array}{l}
\frac{\mu}{i^{2}(1-\alpha)} ; i \leq w \\
\frac{\mu}{w^{2}(1-\alpha)} ; w<i<2 w-1 \\
\frac{\mu}{w^{2}(1-\alpha)} ; i \geq 2 w-1 .
\end{array}\right.
$$

The upper and lower control limits are:

$$
U C L / L C L= \begin{cases}\frac{\mu_{0}}{1-\alpha_{0}} \pm H \sqrt{\sum_{j=1}^{i}\left(\frac{1}{j}\right) \frac{\mu_{0}}{i^{2}\left(1-\alpha_{0}\right)}} & ; i \leq w \\ \frac{\mu_{0}}{1-\alpha_{0}} \pm H \sqrt{\sum_{j=i-w+1}^{i}\left(\frac{1}{j}\right)+(i-w+1)\left(\frac{1}{j}\right) \frac{\mu_{0}}{w^{2}\left(1-\alpha_{0}\right)}} & ; w<i<2 w-1 \\ \frac{\mu_{0}}{1-\alpha_{0}} \pm H \sqrt{\frac{\mu_{0}}{w^{2}\left(1-\alpha_{0}\right)}} & ; i \geq 2 w-1 .\end{cases}
$$

where $H$ is a constant to be chosen.

\subsection{Explicit Formula for Evaluate Average Run Length of Double MovingAverage Control Chart}

Proposition I. Explicit formulas of $A R L_{0}$ for double moving average control chart.

$$
\begin{aligned}
A R L_{0}= & {\left[1-\sum_{i=1}^{w-1}\left[P\left(Z_{1}>\frac{\left(\frac{\mu_{0}}{1-\alpha_{0}}+H \sqrt{\frac{\mu_{0}}{i^{2}\left(1-\alpha_{0}\right)} \sum_{j=1}^{i} \frac{1}{j}}\right)-0.5-\frac{\mu_{0}}{1-\alpha_{0}}}{\sqrt{\frac{\mu_{0}}{i^{2}\left(1-\alpha_{0}\right)} \sum_{j=1}^{i} \frac{1}{j}}}\right]\right.\right.} \\
& \left.\left.+P\left(Z_{1}>\frac{\left(\frac{\mu_{0}}{1-\alpha_{0}}-H \sqrt{\frac{\mu_{0}}{i^{2}\left(1-\alpha_{0}\right)} \sum_{j=1}^{i} \frac{1}{j}}\right)-0.5-\frac{\mu_{0}}{1-\alpha_{0}}}{\sqrt{\frac{\mu_{0}}{i^{2}\left(1-\alpha_{0}\right)} \sum_{j=1}^{i} \frac{1}{j}}}\right)\right]\right]
\end{aligned}
$$


Preprints (www.preprints.org)

| NOT PEER-REVIEWED | Posted: 18 August 2016

doi:10.20944/preprints201608.0169.v1

5 of 11

$$
\begin{aligned}
& -\sum_{j=w+1}^{2 w-2}\left[P\left(Z_{2}>\frac{\frac{\mu_{0}}{1-\alpha_{0}}+H \sqrt{\frac{\mu_{0}}{w^{2}\left(1-\alpha_{0}\right)} \sum_{j=i-w+1}^{w-1} \frac{1}{j}+(i-w+1)\left(\frac{1}{j}\right)}-0.5-\frac{\mu_{0}}{1-\alpha_{0}}}{\sqrt{\frac{\mu_{0}}{w^{2}\left(1-\alpha_{0}\right)} \sum_{j=i-w+1}^{w-1} \frac{1}{j}+(i-w+1)\left(\frac{1}{j}\right)}}\right]\right. \\
& +P\left(Z_{2}<\frac{\frac{\mu_{0}}{1-\alpha_{0}}-H \sqrt{\frac{\mu_{0}}{w^{2}\left(1-\alpha_{0}\right)} \sum_{j=i-w+1}^{w-1} \frac{1}{j}+(i-w+1)\left(\frac{1}{j}\right)}-0.5-\frac{\mu_{0}}{1-\alpha_{0}}}{\sqrt{\frac{\mu_{0}}{w^{2}\left(1-\alpha_{0}\right)} \sum_{j=i-w+1}^{w-1} \frac{1}{j}+(i-w+1)\left(\frac{1}{j}\right)}}\right) \\
& \times P\left(Z_{3}>\frac{\left(\frac{\mu_{0}}{1-\alpha_{0}}+H \sqrt{\frac{\mu_{0}}{w^{2}\left(1-\alpha_{0}\right)}}\right)-0.5-\frac{\mu_{0}}{1-\alpha_{0}}}{\sqrt{\frac{\mu_{0}}{w^{2}\left(1-\alpha_{0}\right)}}}\right) \\
& \left.+P\left(Z_{3}>\frac{\left(\frac{\mu_{0}}{1-\alpha_{0}}-H \sqrt{\frac{\mu_{0}}{w\left(1-\alpha_{0}\right)}}\right)-0.5-\frac{\mu_{0}}{1-\alpha_{0}}}{\sqrt{\frac{\mu_{0}}{w\left(1-\alpha_{0}\right)}}}\right)\right]^{-1}+(2 w-2) .
\end{aligned}
$$

Proposition II. Explicit formulas of $A D T$ for double moving average control chart.

$$
\begin{aligned}
& A D T=\left[1-\sum_{i=1}^{w-1}\left[P\left(Z_{1}>\frac{\left(\frac{\mu_{0}}{1-\alpha_{0}}+H \sqrt{\frac{\mu_{0}}{i^{2}\left(1-\alpha_{0}\right)} \sum_{j=1}^{i} \frac{1}{j}}\right)-0.5-\frac{\mu_{1}}{1-\alpha_{1}}}{\sqrt{\frac{\mu_{1}}{i^{2}\left(1-\alpha_{1}\right)} \sum_{j=1}^{i} \frac{1}{j}}}\right]\right.\right. \\
& \left.\left.+P\left(Z_{1}>\frac{\left(\frac{\mu_{0}}{1-\alpha_{0}}-H \sqrt{\frac{\mu_{0}}{i^{2}\left(1-\alpha_{0}\right)} \sum_{j=1}^{i} \frac{1}{j}}\right)-0.5-\frac{\mu_{1}}{1-\alpha_{1}}}{\sqrt{\frac{\mu_{1}}{i^{2}\left(1-\alpha_{1}\right)} \sum_{j=1}^{i} \frac{1}{j}}}\right)\right]\right] \\
& -\sum_{j=w+1}^{2 w-2}\left[P\left(Z_{2}>\frac{\frac{\mu_{0}}{1-\alpha_{0}}+H \sqrt{\frac{\mu_{0}}{w^{2}\left(1-\alpha_{0}\right)} \sum_{j=i-w+1}^{w-1} \frac{1}{j}+(i-w+1)\left(\frac{1}{j}\right)}-0.5-\frac{\mu_{1}}{1-\alpha_{1}}}{\sqrt{\frac{\mu_{1}}{w^{2}\left(1-\alpha_{1}\right)} \sum_{j=i-w+1}^{w-1} \frac{1}{j}+(i-w+1)\left(\frac{1}{j}\right)}}\right]\right. \\
& +P\left(Z_{2}<\frac{\frac{\mu_{0}}{1-\alpha_{0}}-H \sqrt{\frac{\mu_{0}}{w^{2}\left(1-\alpha_{0}\right)} \sum_{j=i-w+1}^{w-1} \frac{1}{j}+(i-w+1)\left(\frac{1}{j}\right)}-0.5-\frac{\mu_{1}}{1-\alpha_{1}}}{\sqrt{\frac{\mu_{1}}{w^{2}\left(1-\alpha_{1}\right)} \sum_{j=i-w+1}^{w-1} \frac{1}{j}+(i-w+1)\left(\frac{1}{j}\right)}}\right) \\
& \times\left[P\left(Z_{3}>\frac{\left(\frac{\mu_{0}}{1-\alpha_{0}}+H \sqrt{\frac{\mu_{0}}{w^{2}\left(1-\alpha_{0}\right)}}\right)-0.5-\frac{\mu_{1}}{1-\alpha_{1}}}{\sqrt{\frac{\mu_{1}}{w^{2}\left(1-\alpha_{1}\right)}}}\right)\right.
\end{aligned}
$$




$$
\left.+P\left(Z_{3}>\frac{\left(\frac{\mu_{0}}{1-\alpha_{0}}-H \sqrt{\frac{\mu_{0}}{w\left(1-\alpha_{0}\right)}}\right)-0.5-\frac{\mu_{1}}{1-\alpha_{1}}}{\sqrt{\frac{\mu_{1}}{w\left(1-\alpha_{1}\right)}}}\right)\right]^{-1}+(2 w-2)
$$

Proof. The proposition I and II can be analytically derived by central limit theorem as follows. The average run length values can be derived. Let $A R L=n$, then

$$
\begin{aligned}
\frac{1}{A R L}= & \frac{1}{n} P(\text { o.o.c signal at time } i \leq w)+\frac{1}{n} P(\text { o.o.c signal at time } w<i<2 w-1) \\
& +\left[\frac{n-(2 w-2)}{n}\right] P(\text { o.o.c signal at time } i \geq 2 w-1) \\
= & \frac{1}{n}\left\{\sum_{i=1}^{w}\left[P\left(\frac{\sum_{j=1}^{i} M A_{j}}{i}>U C L_{i}\right)+P\left(\frac{\sum_{j=1}^{i} M A_{j}}{i}<L C L_{i}\right)\right]\right\} \\
& +\frac{1}{n}\left\{\sum_{j=i-w+1}^{2 w-2}\left[P\left(\frac{\sum_{j=i-w+1}^{i} M A_{j}}{w}>U C L_{i}\right)+P\left(\frac{\sum_{j=i-w+1}^{i} M A_{j}}{w}<L C L_{i}\right)\right]\right\} \\
& +\left[\frac{n-(2 w-2)}{n}\right]\left(P\left(\frac{\sum_{j=i-w+1}^{i} M A_{j}}{w}>U C L_{w}\right)+P\left(\frac{\sum_{j=i-w+1}^{i} M A_{j}}{w}<L C L_{w}\right)\right) .
\end{aligned}
$$

Let

$$
Z_{1}=\frac{\sum_{j=1}^{i} M A_{j}-\frac{\mu}{1-\alpha}}{\sqrt{\frac{\mu}{i(1-\alpha)} \sum_{j=1}^{i} \frac{1}{j}}}, Z_{2}=\frac{\sum_{j=i-w+1}^{i} M A_{j}-\frac{\mu}{1-\alpha}}{\sqrt{\frac{\mu}{w(1-\alpha)} \sum_{j=i-w+1}^{w-1} \frac{1}{j}+(i-w+1)\left(\frac{1}{j}\right)}} \text { and } Z_{3}=\frac{\sum_{j=1}^{i} M A_{j}-\frac{\mu}{1-\alpha}}{\sqrt{\frac{\mu}{w(1-\alpha)}}} \text {. }
$$

\section{Results}

The numerical results for ARLo and ADT of DMA chart are calculated from Equation (4) and Equation (5). The parameter values for DMA chart following moving average considering $w=2,3$, 4, 5, 10 and 15. The in-control parameter are given $\mu_{0}=1,3$ and $\alpha_{0}=0.2$. Out-of-control parameter values are $\mu_{1}$ and shift parameters $\left(\delta_{1}\right)=0.1,0.2,0.3, \ldots, 2.0$. Out-of-control parameter values are $\alpha_{1}$ and shift parameters $\left(\delta_{2}\right)=0.1,0.2,0.3, \ldots, 2.0$. Out-of-control parameter values are $\mu_{1} / 1-\alpha_{1}$ and shift parameters $(\delta)=0.1,0.2,0.3, \ldots, 2.0$. The results show that the proposed DMA chart are sensitive to only a few of the out-of-control situations.

Table I shows ARL for $\mu_{0}=1$ of DMA chart considering a change in $\mu$, when small shifts $(\delta \leq$ $0.5)$, the DMA chart has the best performance with $w=15$. For moderate shifts $(0.6 \leq \delta \leq 0.8)$ the performance of DMA chart with $w=10$ is superior to others. For the shift sizes $(0.9 \leq \delta \leq 1.0)$ the performance of DMA chart with $w=5$ is the best control chart. For large shifts $(\delta>1.5)$, the DMA chart has the best performance with $w=4$. Table II shows the ARL for $\mu_{0}=3$ of DMA chart considering a change in $\mu$, when small shifts $(\delta \leq 0.3)$ the DMA chart has the best performance with $w=15$. For moderate shifts $(0.4 \leq \delta \leq 0.5)$, the DMA chart has good performance with $w=10$. For magnitude of shifts $(0.6 \leq \delta \leq 0.7)$, the DMA chart has good performance with $w=5$. For parameter shift $(0.8 \leq \delta \leq 0.9)$, the DMA chart has the performance with $w=4$. For parameter shifts $(\delta \leq 1.0)$, the DMA chart has good performance with $w=3$. For large shifts $(\delta>1.5)$, the DMA chart has good performance with $w=2$. 
Table 1. ARL of DMA chart for given $\mu_{0}=1, \alpha_{0}=0.2$ and considering a change in $\mu$.

\begin{tabular}{ccccccc}
\hline$\delta_{1}$ & $w=\mathbf{2}$ & $w=\mathbf{3}$ & $w=\mathbf{4}$ & $w=\mathbf{5}$ & $w=\mathbf{1 0}$ & $w=\mathbf{1 5}$ \\
\hline 0.0 & 370.398 & 370.398 & 370.398 & 370.398 & 370.398 & 370.398 \\
0.1 & 201.525 & 173.237 & 144.981 & 119.819 & 52.267 & $37.871^{*}$ \\
0.2 & 101.168 & 70.595 & 49.631 & 36.400 & $20.347^{*}$ & 25.745 \\
0.3 & 53.518 & 32.788 & 21.917 & 16.632 & $16.182^{*}$ & 22.985 \\
0.4 & 30.909 & 17.946 & 12.51 & $10.627^{*}$ & 14.537 & 19.277 \\
0.5 & 19.475 & 11.361 & 8.696 & $8.296^{*}$ & 12.952 & 15.033 \\
0.6 & 13.253 & 8.086 & $6.879^{*}$ & 7.160 & 11.161 & 11.468 \\
0.7 & 9.629 & 6.290 & $5.883^{*}$ & 6.468 & 9.375 & 8.942 \\
0.8 & 7.389 & $5.219^{*}$ & 5.261 & 5.956 & 7.824 & 7.273 \\
0.9 & 5.933 & $4.530^{*}$ & 4.824 & 5.519 & 6.593 & 6.163 \\
1.0 & 4.943 & $4.057^{*}$ & 4.482 & 5.116 & 5.655 & 5.382 \\
1.5 & $2.816^{*}$ & 2.894 & 3.272 & 3.443 & 3.355 & 3.347 \\
2.0 & $2.126^{*}$ & 2.307 & 2.450 & 2.461 & 2.434 & 2.434 \\
\hline \multicolumn{7}{c}{ is minimum ADT. }
\end{tabular}

Table 2. ARL of DMA chart for given $\mu_{0}=3, \alpha_{0}=0.2$ and considering a change in $\mu$.

\begin{tabular}{ccccccc}
\hline$\delta_{1}$ & $w=\mathbf{2}$ & $w=\mathbf{3}$ & $w=\mathbf{4}$ & $w=\mathbf{5}$ & $w=\mathbf{1 0}$ & $w=\mathbf{1 5}$ \\
\hline 0.0 & 370.398 & 370.398 & 370.398 & 370.398 & 370.398 & 370.398 \\
0.1 & 154.065 & 109.625 & 77.476 & 56.092 & $24.402^{*}$ & 27.203 \\
0.2 & 54.704 & 30.006 & 18.952 & $14.307^{*}$ & 15.693 & 21.742 \\
0.3 & 23.298 & 12.253 & 8.912 & $8.391^{*}$ & 12.850 & 14.187 \\
0.4 & 12.087 & 7.014 & $6.229^{*}$ & 6.745 & 9.475 & 8.874 \\
0.5 & 7.399 & $5.020^{*}$ & 5.146 & 5.837 & 6.828 & 6.369 \\
0.6 & 5.153 & $4.075^{*}$ & 4.502 & 5.064 & 5.217 & 5.066 \\
0.7 & 3.949 & $3.529^{*}$ & 3.996 & 4.347 & 4.250 & 4.217 \\
0.8 & 3.239 & $3.154^{*}$ & 3.548 & 3.721 & 4.250 & 3.602 \\
0.9 & $2.785^{*}$ & 2.861 & 3.148 & 3.213 & 3.138 & 3.137 \\
1.0 & $2.472^{*}$ & 2.615 & 2.801 & 2.816 & 2.776 & 2.776 \\
1.5 & $1.702^{*}$ & 1.772 & 1.778 & 1.778 & 1.777 & 1.777 \\
2.0 & $1.356^{*}$ & 1.366 & 1.366 & 1.366 & 1.366 & 1.366 \\
\hline \multicolumn{7}{c}{ is minimum ADT. }
\end{tabular}

Table III shows the ARL for $\mu_{0}=1$ of DMA chart considering a change in $\alpha$. For small shifts, ( $\delta$ $\leq 0.4)$ the DMA chart has the best performance with $w=15$. For moderate shifts $(0.5 \leq \delta \leq 0.8)$, the DMA chart has good performance with $w=10$. For magnitude of shifts $(0.9 \leq \delta \leq 1.0)$, the DMA chart has good performance with $w=5$. For parameter shift $(\delta \leq 1.5)$, the DMA chart has the performance with $w=4$. For parameter shifts $(\delta \leq 2.0)$, the DMA chart has good performance with $w=3$. Table IV shows the ARL for $\mu_{0}=3$ of DMA chart considering a change in $\alpha$, when small shifts, $(\delta \leq 0.3)$, the DMA chart has the best performance with $w=15$. For moderate shifts $(0.4 \leq \delta \leq 0.5)$, the DMA chart has good performance with $w=10$. For magnitude of shifts $(0.6 \leq \delta \leq 0.9)$, the DMA chart has good performance with $w=5$. For parameter shift $(\delta \leq 1.0)$, the DMA chart has the performance with $w=$ 
4. For parameter shifts $(\delta \leq 1.5)$, the DMA chart has good performance with $w=3$. For parameter shifts $(\delta \leq 2.0)$, the DMA chart has good performance with $w=2$.

Table 3. ARL of DMA chart for given $\mu_{0}=1, \alpha_{0}=0.2$ and considering a change in $\alpha$.

\begin{tabular}{ccccccc}
\hline$\delta_{2}$ & $w=\mathbf{2}$ & $w=\mathbf{3}$ & $w=\mathbf{4}$ & $w=5$ & $w=\mathbf{1 0}$ & $w=\mathbf{1 5}$ \\
\hline 0.0 & 370.398 & 370.398 & 370.398 & 370.398 & 370.398 & 370.398 \\
0.1 & 298.968 & 284.152 & 273.606 & 264.593 & 221.813 & $180.796^{*}$ \\
0.2 & 237.34 & 210.69 & 190.885 & 173.909 & 109.624 & $75.241^{*}$ \\
0.3 & 185.959 & 152.819 & 129.232 & 110.495 & 57.390 & $42.074^{*}$ \\
0.4 & 144.351 & 109.707 & 86.925 & 70.495 & 34.932 & $31.206^{*}$ \\
0.5 & 111.428 & 78.711 & 59.079 & 46.243 & $24.896^{*}$ & 27.102 \\
0.6 & 85.822 & 56.855 & 41.020 & 31.608 & $20.087^{*}$ & 25.202 \\
0.7 & 66.141 & 41.565 & 29.296 & 22.663 & $17.577^{*}$ & 24.022 \\
0.8 & 51.125 & 30.867 & 21.607 & 17.080 & $16.119^{*}$ & 23.006 \\
0.9 & 39.711 & 23.344 & 16.488 & $13.506^{*}$ & 15.151 & 21.917 \\
1.0 & 31.043 & 18.010 & 13.02 & $11.155^{*}$ & 14.401 & 20.649 \\
1.5 & 10.335 & 6.733 & $6.141^{*}$ & 6.547 & 10.763 & 12.284 \\
2.0 & 4.494 & $3.863^{*}$ & 4.264 & 4.893 & 6.494 & 6.268 \\
\hline
\end{tabular}

* is minimum ADT.

Table 4. ARL of DMA chart for given $\mu_{0}=3, \alpha_{0}=0.2$ and considering a change in $\alpha$.

\begin{tabular}{ccccccc}
\hline$\delta_{2}$ & $w=\mathbf{2}$ & $w=\mathbf{3}$ & $w=\mathbf{4}$ & $w=\mathbf{5}$ & $w=\mathbf{1 0}$ & $w=\mathbf{1 5}$ \\
\hline 0.0 & 370.398 & 370.398 & 370.398 & 370.398 & 370.398 & 370.398 \\
0.1 & 291.784 & 270.623 & 252.542 & 235.367 & 158.437 & $108.104^{*}$ \\
0.2 & 217.048 & 178.177 & 147.713 & 122.847 & 56.437 & $40.275^{*}$ \\
0.3 & 155.32 & 111.86 & 83.083 & 63.430 & 28.700 & $28.590^{*}$ \\
0.4 & 108.909 & 69.874 & 48.025 & 35.266 & $20.289^{*}$ & 25.573 \\
0.5 & 75.922 & 44.520 & 29.402 & 21.751 & $17.190^{*}$ & 24.007 \\
0.6 & 53.155 & 29.316 & 19.305 & $14.952^{*}$ & 15.698 & 22.378 \\
0.7 & 37.625 & 20.09 & 13.635 & $11.330^{*}$ & 14.673 & 20.276 \\
0.8 & 27.043 & 14.375 & 10.322 & $9.276^{*}$ & 13.707 & 17.730 \\
0.9 & 19.794 & 10.748 & 8.300 & $8.028^{*}$ & 12.644 & 15.033 \\
1.0 & 14.783 & 9.385 & $7.010^{*}$ & 7.207 & 11.447 & 12.510 \\
1.5 & 4.772 & $3.944^{*}$ & 4.362 & 4.963 & 5.729 & 5.438 \\
2.0 & $2.479^{*}$ & 2.638 & 2.935 & 3.071 & 3.032 & 3.018 \\
\hline
\end{tabular}

${ }^{*}$ is minimum ADT.

Table V shows the ARL for $\mu_{0}=1$ and $\alpha_{0}=0.2$ of DMA chart considering a change in $\mu / 1-\alpha$. For small shifts $(\delta \leq 0.5)$, the DMA chart has the best performance with $w=15$. For moderate shifts $(0.6 \leq \delta \leq 0.8)$, the DMA chart has good performance with $w=10$. For magnitude of shifts $(\delta \leq 0.9)$, the DMA chart has good performance with $w=5$. For parameter shift $(1.0 \leq \delta \leq 1.5)$, the DMA chart has the performance with $w=4$. For parameter shifts $(\delta \leq 2.0)$, the DMA chart has good performance with $w=3$. Table VI shows the ARL for $\mu_{0}=3$ and $\alpha_{0}=0.2$ of DMA chart considering a change in 
$\mu / 1-\alpha$, when small shifts $(\delta \leq 0.3)$, the DMA chart has the best performance with $w=15$. For moderate shifts $(0.4 \leq \delta \leq 0.5)$, the DMA chart has good performance with $w=10$. For magnitude of shifts $(0.6 \leq \delta \leq 0.7)$, the DMA chart has good performance with $w=5$. For parameter shift $(0.8 \leq \delta \leq$ $0.9)$, the DMA chart has the performance with $w=4$. For parameter shifts $(\delta \leq 1.0)$, the DMA chart has good performance with $w=3$. For parameter shifts $(1.5 \leq \delta \leq 2.0)$, the DMA chart has good performance with $w=2$.

The average run length for DMA chart shows that when the shift increases, the DMA performs better when the value of $(w)$ decreases. Therefore, one can see that the proposed formulas for ARLo and ADT for DMA chart can correctly calculate efficiently. Moreover, this is easy to implement which should also greatly reduce computation times.

Table 5. ARL of DMA chart for given $\mu_{0}=1, \alpha_{0}=0.2$ and considering a change in $\mu /(1-\alpha)$.

\begin{tabular}{ccccccc}
\hline$\delta$ & $w=\mathbf{2}$ & $w=\mathbf{3}$ & $w=\mathbf{4}$ & $w=\mathbf{5}$ & $w=\mathbf{1 0}$ & $w=\mathbf{1 5}$ \\
\hline 0.0 & 370.398 & 370.398 & 370.398 & 370.398 & 370.398 & 370.398 \\
0.1 & 329.399 & 311.906 & 296.057 & 281.64 & 225.731 & $187.864^{*}$ \\
0.2 & 244.313 & 206.88 & 178.542 & 156.438 & 94.298 & $67.119^{*}$ \\
0.3 & 166.124 & 126.463 & 100.728 & 82.916 & 42.669 & $30.139^{*}$ \\
0.4 & 110.348 & 77.170 & 58.018 & 45.897 & 22.805 & $17.995^{*}$ \\
0.5 & 73.723 & 48.334 & 34.988 & 27.154 & 14.518 & $13.397^{*}$ \\
0.6 & 50.111 & 31.352 & 22.263 & 17.293 & $10.722^{*}$ & 11.312 \\
0.7 & 34.805 & 21.130 & 14.989 & 11.872 & $8.7909^{*}$ & 10.111 \\
0.8 & 24.743 & 14.816 & 10.682 & 8.756 & $7.677^{*}$ & 9.206 \\
0.9 & 18.017 & 10.811 & 8.041 & $6.883^{*}$ & 6.931 & 8.372 \\
1.0 & 13.441 & 8.204 & 6.363 & $5.704^{*}$ & 6.349 & 7.538 \\
1.5 & 4.424 & 3.389 & $3.282^{*}$ & 3.407 & 3.986 & 4.057 \\
2.0 & 2.377 & $2.263^{*}$ & 2.359 & 2.439 & 2.506 & 2.506 \\
\hline \multicolumn{7}{c}{ is minimum ADT. }
\end{tabular}

Table 6. ARL of DMA chart for given $\mu_{0}=3, \alpha_{0}=0.2$ and considering a change in $\mu /(1-\alpha)$.

\begin{tabular}{ccccccc}
\hline$\delta$ & $w=\mathbf{2}$ & $w=\mathbf{3}$ & $w=\mathbf{4}$ & $w=\mathbf{5}$ & $w=\mathbf{1 0}$ & $w=\mathbf{1 5}$ \\
\hline 0.0 & 370.398 & 370.398 & 370.398 & 370.398 & 370.398 & 370.398 \\
0.1 & 267.918 & 234.024 & 207.107 & 185.277 & 119.142 & $87.114^{*}$ \\
0.2 & 137.471 & 100.337 & 77.601 & 62.546 & 31.204 & $22.948^{*}$ \\
0.3 & 68.24 & 44.271 & 31.88 & 24.707 & 13.538 & $12.866^{*}$ \\
0.4 & 35.700 & 21.709 & 15.392 & 12.167 & $8.894^{*}$ & 10.183 \\
0.5 & 20.013 & 11.979 & 8.8039 & 7.421 & $7.159^{*}$ & 8.654 \\
0.6 & 12.061 & 7.439 & 5.877 & $5.362^{*}$ & 6.1427 & 7.208 \\
0.7 & 7.814 & 5.151 & 4.432 & $4.326^{*}$ & 5.284 & 5.813 \\
0.8 & 5.428 & 3.909 & $3.633^{*}$ & 3.708 & 4.476 & 4.655 \\
0.9 & 4.023 & 3.181 & $3.133^{*}$ & 3.270 & 3.754 & 3.795 \\
1.0 & 3.157 & $2.719^{*}$ & 2.778 & 2.912 & 3.165 & 3.171 \\
1.5 & $1.621^{*}$ & 1.664 & 1.689 & 1.694 & 1.695 & 1.695 \\
2.0 & $1.192^{*}$ & 1.197 & 1.198 & 1.198 & 1.198 & 1.198 \\
\hline \multicolumn{7}{c}{ is minimum ADT. }
\end{tabular}

* is minimum ADT. 


\section{Discussion}

The best properties of a DMA chart are memory control charts because of their ability to detect small shifts. Without loss of generality, this chart can be relaxed due to its feasibility with the width of control limit $(w)$. The DMA chart performs better as the values of $w$ increases for small shifts, however, the number of observations must be sufficiently large.

\section{Conclusions}

The explicit formulas for ARL of DMA for Poisson counting process was derived. The INAR(1) model is a simple but well interpretable model for correlated process of Poisson counts data. The result shows that when a process increases, the performance of DMA will perform better as the value of $w$ decreases for all case studies. Furthermore, these explicit formulas are simple and easy to implement which reduces computation time to less than 1 second.

Acknowledgments: This research is granted from National Research Council of Thailand with contract no. KMUTNB-GOV-60-XX. The author would like to express gratitude to the Graduate Collage of King Mongkut's University of Technology, North Bangkok, Thailand. Also thanks to the Department of Applied Statistics for supporting materials and high performance computer.

Conflicts of Interest: The authors declare no conflict of interest.

\section{References}

1. Harris, T.E. 1963. The Theory of Branching Processes, Springer, Berlin, 1963.

2. Wei $\beta$, C.H. Controlling correlated processes of Poisson counts. Quality Reliability Engineering International 2007, 23, 741-754.

3. Montgomery, D.C. Statistical Quality Control, 6th ed.; John Wiley \& Sons, New York, 2009.

4. Roberts, S.W. Control chart tests based on geometric moving average. Technometrics 1959, 1, $239-250$.

5. Page, E.S. Continuous inspection schemes. Biometrika 1954, 41, 100-144.

6. Khoo, M.B.C. A moving average control chart for monitoring the fraction non-conforming. Journal of Quality and Reliability Engineering International 2004, 20, 617-635.

7. Khoo, M.B.C; Wong, V.H. A double moving average control chart. Communications in Statistics-Simulation and Computation 2008, 37, 1696-1708.

8. Brockwell, P.J.; Davis, R.A. Time Series: Data Analysis and theory, 2nd ed.; Springer, New York, 2009.

9. McKenzie, E. Some simple models for discrete variate time series. Water Resources Bulletin 1985, 21, 645-650.

10. Al.Osh, M.A.; Alzaid, A.A. First-order integer-valued autoregressive (INAR(1)) process. Journal of Time Series Analysis 1987, 8, 261-275.

11. McKenzie, E. Discrete Variate Time Series in Handbook of statistics eds, Shanbhag, D.N., Rao, C.R., Elsevier Amsterdam, 2003; volume 20, pp. 573-606.

12. Crowder, S.V. A simple method for studying run length distributions of exponentially weighted moving average charts. Technometrics 1979, 29, 401-407.

13. Brook, D.; Evans, D.A. An approach to the probability distribution of Cusum run length. Biometrika 1972, 9, 539-548.

14. Areepong, Y.; Novikov, A.A. Martingale approach to EWMA control chart for changes in Exponential distribution. Journal of Quality Measurement and Analysis 2008, 4, 197-203.

15. Areepong, Y.; Sukparungsee, S. An analytical ARL of binomial double moving average chart. International Journal of Pure and Applied Mathematics 2011, 73, 477-488.

16. Areepong, Y. Explicit formulas of Average Run Length for a Moving Average control chart for monitoring the number of defective products. International Journal of Pure and Applied Mathematics 2012, 80, 331-343.

17. Areepong, Y.; Sukparungsee, S. Closed form formulas of average run length of moving average control chart for nonconforming for zero-inflated process. Far East Journal of Mathematical Sciences 2013, 75, 385-400.

18. Sukparungsee, S. Average run length of double moving average control chart for zero-inflated count processes. Far East Journal of Mathematical Sciences 2013, 1, 85-103.

19. Sukparungsee, S.; Areepong, Y. Explicit Expression for the Average Run Length of Double Moving Average Scheme for Zero-Inflated Binomial Process. International Journal of Applied Mathematics E Statisitcs 2015, 53, 33-43. 
20. Phantu, S.; Sukparungsee, S.; Areepong, Y. Explicit expressions of average run length of moving average control chart for poisson integer valued autoregressive model. Lecture Notes in Engineering and Computer Science 2016, 2, 892-895.

21. Steutel, F.W.; Harn, K.V. Discrete analogues of self decomposability and stability. Annals of Probability 1979, 7, 839-899.

(C) 2016 by the authors; licensee Preprints, Basel, Switzerland. This article is an open access article distributed under the terms and conditions of the Creative Commons by Attribution (CC-BY) license (http://creativecommons.org/licenses/by/4.0/). 